\title{
Saint Jérôme et la Vie de Panl de Thèbes.
}

M. Bidez a publié en 1900 deux Vies grecques inédites de Paul de Thèbes ${ }^{1}$ ), et a démontré que ces Vies grecques étaient des versions de la Vie latine écrite par saint Jérôme: que l'une d'elles, $a$, était une traduction littérale du texte latin, et l'autre, $b$, un remaniement, un abrégé de $a$. M. Nau a contesté les conclusions de M. Bidez dans les Analecta Bollandiana ${ }^{2}$ ), et s'est efforcé d'établir, en vain selon nous, que la Vie latine de Paul de Thèbes serait une traduction libre de $b$, et $a$ une revision de $b$ sur le latin, revision faite à une époque relativement moderne par un moine helléniste. Sans vouloir reprendre la discussion de cette question, nous nous proposons, dans le présent article, de faire valoir un argument nouveau, et assez important, nous semble-t-il, en faveur de la thèse de $M$. Bidez.

M. Nau a très bien fait ressortir dans son travail que l'auteur de la Vie de Paul de Thèbes a imité, à maintes reprises, la Vie d'Antoine par saint Athanase. ${ }^{3}$ ) Retrouvant dans la rédaction grecque $b$ les mots eux-mêmes dont saint Athanase s'est servi, M. Nau voit dans ce fait, qu'il exagère d'ailleurs un peu, une des preuves directes de la priorité de $b$, et il estime que cette preuve ne peut pas être utilisée dans une autre hypothèse que la sienne. M. Van den Ven a déjà signalé la faiblesse de cet argument ${ }^{4}$ ): il a fait remarquer que les réminiscences de la Vie de saint Antoine dans la rédaction $b$ «n'ont rien qui doivent étonner, quand on songe à l'énorme sucè̀s de l'écrit de saint Athanase dans les milieux monastiques», de plus que le traducteur de la Vie d'Hilarion de Gaza, comme le rédacteur $b$ de la Vie de Paul de Thèbes, est souvent plus près de la Vie d'Antoine que l'auteur (c'est-à-dire saint Jérôme) lui-même. Nous allons pour notre

1) Deux versions grecques inédites de la Vie de Paul de Thèbes, Gand, 1900 , in- $8^{\circ}$.

2) T. XX (1901) p. 121-157 (Le texte grec original de la Vie de S. Paul de Thèbes).

3) La chose n'avait pourtant pas échappé à M. Bidez, mais il a eu tort de n'en parler qu'incidemment en note. ('f. Deux versions etc. p. XLII note 4.

4) S. Jérôme et la Vie du moine Malchus le Captif, Louvain, 1901, in-80, p. 102 , note 2 et p. 132 note 2 . 
part montrer que la preuve en question peut parfaitement être employée dans une autre hypothèse que celle de $M$. Nau.

Nous reconnaissons volontiers que la Vie de Paul de Thèbes est faite sur le plan, sur le patron de la Vie d'Antoine, mais nous estimons qu'elle a été composée, non pas par le rédacteur $b$ d'après le texte grec d'Athanase, mais par saint Jérôme d'après la version latine d'Évagrius.

Saint Jérôme fait allusion dans le prologue de la Vie de Paul de Thèbes à une traduction latine de la Vie de saint Antoine. ${ }^{1}$ ) Cette traduction est sans aucun doute celle d'Évagrius, que tout le monde considère comme antérieure à la Vie de Paul de Thèbes. ${ }^{2}$ ) Il est d'ailleurs tout naturel que saint Jérôme ait connu la version d'Évagrius à l'époque où il composa la Vie du premier anachorète. Il écrivit cette Vie pendant son séjour dans le désert de Chalcis (à l'est d'Antioche), où il ne cessait d'entretenir des relations avec Évagrius, alors encore simple prêtre antiochéen. ${ }^{3}$ ) Ajoutons que plus tard saint Jérôme mentionnera la version d'Éragrius au chapitre CXXV de son Liber de viris illustribus. ${ }^{4}$ )

Nous ne relèverons pas tous les emprunts faits par saint Jérôme à la version d'Évagrius. Quelques exemples suffiront pour établir que saint Jérôme s'en est inspiré, et que c'est bien sa rédaction qui est la rédaction primitive de la Vie de Paul de Thèbes.

\section{I}

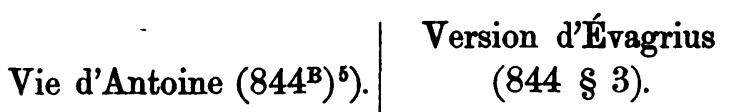

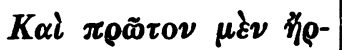

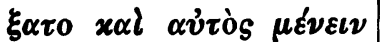

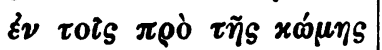

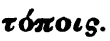

Et primo quidem incipiens etiam ipse in et secretiorem secessit ou locis paululum a villa remotioribus manebat.

1) Migne, Patr. lat., t. XXIII, col. 18: Igitur quia de Antonio tam Graeco quam Romano stylo diligenter memoriae traditum est etc.

2) Bardenhewer place la date de la composition de la Vie de Paul en 376, d'autres en 374 ou 375. Cf. Bidez, Deux versions etc., p. VI. - D'après Batiffol (La littérature grecque chrétienne, Paris, $1898,2^{\mathrm{e}}$ édit. p. 252) la version d'Évagrius a été faite à Verceil entre 363 et 370.

3) Saint Jérôme vécut dans le désert de Chalcis de 374 jusqu'en 379 environ. Cf. Bardenhewer, Patrologie, p. 427; trad. Godet et Verschaffel, t. II, p. 365 . Sur les relations d'amitié de saint Jérôme avec Évagrius, cf. Grützmacher, Hieronymus, Leipzig, 1901, p. 142 et suiv.

4) Migne, Patr. lat., t. XXIII, col. 751.

5) Les renvois visent pour la Vie d'Antoine et la version d'Évagrius le t. XXVI 
Traduction $a(8,3)$.

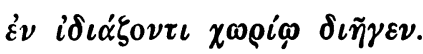

Traduction $b(9,4-5)$.

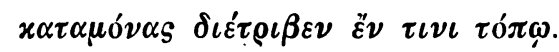

Il est manifeste que les mots de saint Jérôme: in villa remotiore secretior manebat - telle est la bonne leçon, cf. la version d'Évagrius et la traduction $a$ - sont imités de ceux d'Évagrius: in locis paululum a villa remotioribus manebat. Le traducteur a laissant le mot secretior de côté (peut-être ce mot manquait-il dans le texte qu'il avait sous les yeux), a rendu fidèlement in villa remotiore manebat par $\varepsilon^{2} \nu ~ i \delta \iota \alpha \dot{\xi} \xi \nu \tau \iota$

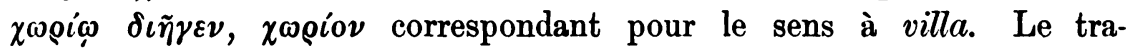
ducteur $b$ donnant à $\chi \omega \varrho i o v$ la signification de «lieu, endroit» a trans-

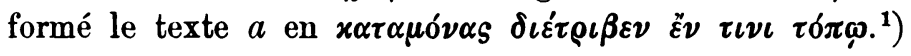

\section{II}

\begin{tabular}{|c|c|c|}
\hline Vie d'Antoine $\left(916^{\mathrm{A}}\right)$. & $\begin{array}{l}\text { Version d'Évagrius } \\
\quad(916, \S 50) .\end{array}$ & Saint Jérôme $\left(21^{\mathrm{B}}\right)$. \\
\hline 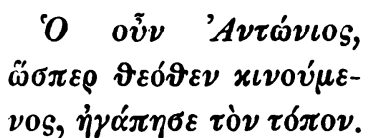 & $\begin{array}{l}\text { Hunc Antonius locum, } \\
\text { quasi a Deo sibi offer- } \\
\text { retur, amplexus est. }\end{array}$ & $\begin{array}{l}\text { Igitur adamato (quasi } \\
\text { quod a Deo sibi offer- } \\
\text { retur) habitaculo. }{ }^{2} \text { ) }\end{array}$ \\
\hline
\end{tabular}

Traduction $a(10,1-2)$.

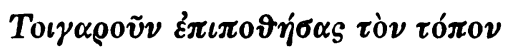

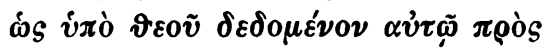

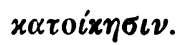

Traduction $b(11,1-2)$.

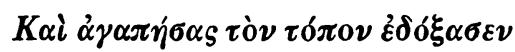

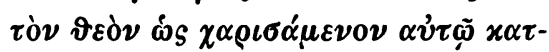

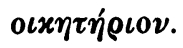

Cet exemple est des plus significatifs. Il indique non seulement avec évidence que saint Jérôme s'est inspiré de la version d'Évagrius mais il montre encore que la rédaction $b$ dérive de la Vie latine. On remarquera en effet que les mots de la version d'Évagrius: quasi a Deo sibi offerretur, qui sont une traduction libre des mots de la Voie d'An-

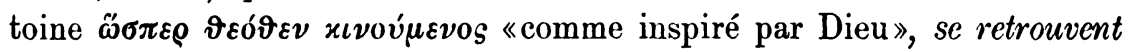

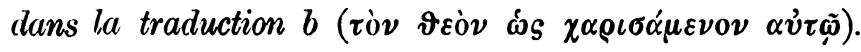

de la Patrologie grecque de Migne, pour le lexte de saint Jérôme le $t$. XXIII de la Palrologie latine, pour les traductions $a$ et $b$ l'ouvrage de M. Bidez: Deux versiows grecques etc.

:) Le mot $\tau \delta ́ \pi \omega$ de la traduction $b$ pouvant aussi être expliqué par la Vie d'Antcine, nous royons uniquement dans ce premier exemple une preuve que saint Jérôme a utilisé la version d'Évagrius.

‡) Le mot habitaculum revient souvent dans la rersion d'Évagrius. 


\section{III}

Vie d'Antoine $\left(920^{\mathrm{A}}\right)$.

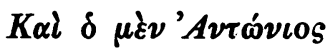

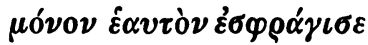

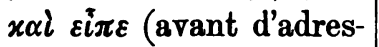
ser la parole à la bête, moitié homme, moitié âne, qu'il vient de voir, Antoine fait le signe de la croix).

Traduction $a$.

\section{Passage omis.}

\section{Version d'Évagrius \\ (920, § 53).}

Post cujus aspectum, vexillum crucis in fronte pingens, hoc tantum ait. fro

\section{Traduction $b$.}

Passage omis.

L'omission, dans les rédactions grecques $a$ et $b$, de ce passage parallèle entre la traduction latine de la Vie d'Antoine et le texte latin de la Vie de Paul de Thèbes, établit d'une façon péremptoire que saint Jérôme s'est servi de la version d'Évagrius. Elle plaide encore en faveur de l'antériorité du texte latin: si, comme le veut $M$. Nau, $b$ est le texte original et $a$ une revision de $b$ faite sur le latin, il faut admettre $1^{\circ}$ que saint Jérôme a complété $b$ au moyen de la version d'Évagrius; $2^{\circ}$ que le reviseur $b$ "qui a travaillé à rétablir le latin» n'a pas jugé à propos de traduire le passage ajouté par saint Jérôme. Or, une double supposition de ce genre est bien peu vraisemblable. Par contre, si l'on admet avec M. Bidez que le texte latin est le texte original, l'omission du passage en question dans les deux rédactions grecques s'explique tout naturellement: $b$ ne présente pas ce passage, parce que $a$ a oublié de le traduire.

\section{IV}

\begin{tabular}{|c|c|c|}
\hline & $\begin{array}{l}\text { Version d'Évagrius } \\
(916, \S 50) \text {. }\end{array}$ & Saint Jérôme $\left(\mathbf{2 7}^{\mathrm{C}}\right)$. \\
\hline 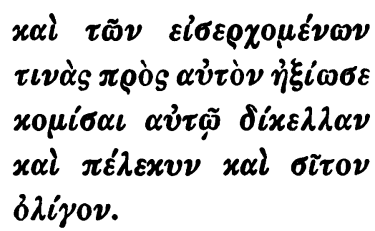 & $\begin{array}{l}\text { rogavit unum de adve- } \\
\text { nientibus, ut sarculum } \\
\text { sibi bis acutum cum } \\
\text { frumento deferret. }\end{array}$ & $\begin{array}{l}\text { contristabatur Antonius, } \\
\text { quod sarculum, quo ter- } \\
\text { ram foderet, non haberet. }\end{array}$ \\
\hline
\end{tabular}

Traduction $a(28,13-15)$.

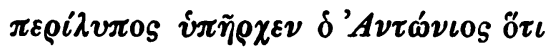

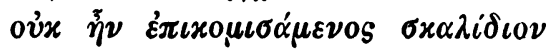

Traduction $b(29,12-13)$.

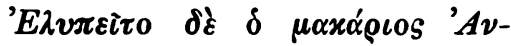

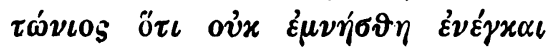




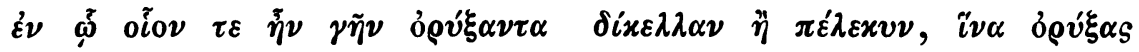
$x \alpha \tau \alpha \vartheta \varepsilon \dot{\sigma} \boldsymbol{\alpha} \iota$.

$\vartheta \alpha^{\prime} \psi \omega$ rò $\sigma \tilde{\omega} \mu \alpha$.

La version d'Évagrius explique pourquoi il n'est question dans la Vie latine de Paul de Thèbes que d'un sarculum: c'est le seul outil que saint Jérôme ait trouvé mentionné chez Évagrius. L'auteur de la traduction $b$, qui connaît bien la Vie d'Antoine, a substitué d'après elle les mots $\delta i x \varepsilon \lambda \lambda \alpha \nu$ et $\pi \dot{\varepsilon} \lambda \varepsilon x v \nu$ au mot $\sigma x \alpha \lambda i \delta \iota 0 \nu$ de la traduction $a .{ }^{1}$ )

\section{V}

L'épilogue, propre à la version d'Évagrius, a enfin été imité par saint Jérôme, et cet épilogue se retrouve dans les deux traductions grecques. $^{2}$ )

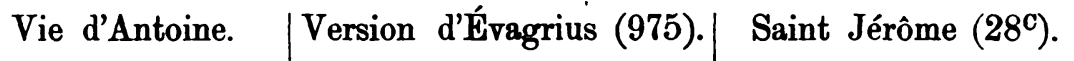
Itaque prudentes qui le- Obsecro, quicumque gere voluerint hanc scriptu- haec legis, ut Hieronymi ram, obsecramus, ut dent peccatoris memineris....
veniam ...

Traduction $a(32,10)$.

Traduction $b(33,12)$.

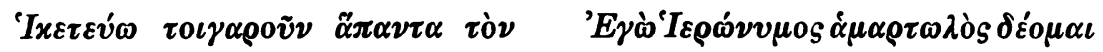

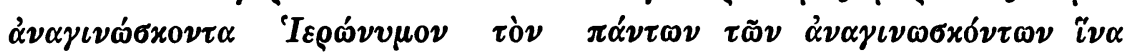

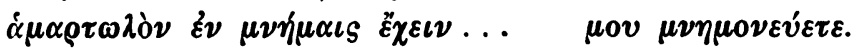

Ill ressort de ces quelques passages parallèles entre la Vie latine de Paul de Thèbes et la version d'Évagrius que saint Jérôme s'est manifestement inspiré de cette dernière. Le fait, à notre avis, ne peut pas être contesté quelque soit le point de vue auquel on se place. La rédaction grecque $b$ présentant dans deux passages $\left(n^{\circ} 2\right.$ et $n^{\circ} 5$ ) des particularités propres à la version d'Evagrius - que son auteur n'a certainement pas connue - il est impossible de conclure à la priorité de $b$. La présence de ces particularités dans $b$ ne peut s'expliquer que si l'on admet, comme M. Bidez l'a démontré par d'autres arguments que le nôtre, que saint Jérôme est le auteur de la première Vie de Paul de Thèbes.

\section{A. Kugener.}

1) Cette substitution ne nous semble pas très heureuse. Antoine n'avait pas besoin d'une hache pour enterrer Paul de Thèbes.

2) Ce passage parallèle entre la version d'Évagrius et la Vie latine de Paul de Thèbes avait déjà été remarqué par M. Bidez, cf. Deux versions grecques etc. p. XLII, note 4. 